Correction

\title{
Correction: Gušić, D. Prime Geodesic Theorems for Compact Locally Symmetric Spaces of Real Rank One. Mathematics 2020, 8,1762
}

\section{Dženan Gušić (D)}

check for updates

Citation: Gušić, D. Correction: Gušić,

D. Prime Geodesic Theorems for Compact Locally Symmetric Spaces of Real Rank One. Mathematics 2020, 8, 1762. Mathematics 2021, 9, 710. https://doi.org/10.3390/math9070710

Received: 19 February 2021 Accepted: 22 February 2021 Published: 25 March 2021

Publisher's Note: MDPI stays neutral with regard to jurisdictional claims in published maps and institutional affiliations.

Copyright: (C) 2021 by the author. Licensee MDPI, Basel, Switzerland. This article is an open access article distributed under the terms and conditions of the Creative Commons Attribution (CC BY) license (https:/ / creativecommons.org/licenses/by/ $4.0 /)$.
Department of Mathematics, University of Sarajevo, Zmaja od Bosne 33-35, 71000 Sarajevo, Bosnia and Herzegovina; dzenang@pmf.unsa.ba

The author wishes to make the following correction to the paper [1].

It was found that there was a typo in the abstract section of [1]. Namely, it should be $O\left(x^{2 \rho-\frac{\rho}{n}}(\log x)^{-1}\right)$ in place of $O\left(x^{2 \rho-\frac{\rho}{\eta}}(\log x)^{-1}\right)$. This change has no material impact on the conclusions of the paper.

In [1], we derived a number of results on prime geodesic theorems for compact, even-dimensional, locally symmetric Riemannian manifolds of strictly negative sectional curvature. For the sake of readers and the overall completeness of the research, we would also like to complement [1] with analogous results in odd dimensions. Thus, we shall briefly prove that the results obtained in [1] remain valid if the dimension of the underlying locally symmetric space is assumed to be odd.

Let $Y$ be a compact, $n$-dimensional ( $n$ odd), locally symmetric Riemannian manifold of strictly negative sectional curvature. The rest of the notation used below will be fully adopted from [1].

The following results hold true (see, [1] (Theorems 1-3) for the counterparts in the even-dimensional case).

Theorem 1. Let $Y$ be as above. Then

$$
\psi_{0}(x)=\sum_{p=0}^{n-1}(-1)^{p+1} \sum_{(\tau, \lambda) \in I_{p}} \sum_{\substack{\alpha \in S_{p, \tau, \lambda}^{\mathbb{R}} \\ 2 \rho-\frac{\rho}{n}<\alpha \leq 2 \rho}} \alpha^{-1} x^{\alpha}+O\left(x^{2 \rho-\frac{\rho}{n}}\right),
$$

where $S_{p, \tau, \lambda}^{\mathbb{R}}$ denotes the set of real singularities of $Z_{S}(s+\rho-\lambda, \tau)$.

Proof. We adjust the proof of Theorem 1 in [1] (pp. 6-9).

The singularities of $Z_{S}(s+\rho-\lambda, \tau)$ are given by Theorem 3.15 in [2] (pp. 113-115).

Since $n$ is odd, there are only spectral singularities, so the part of the proof related to topological singularities is missing now. The actual proof (in odd dimensions) is therefore much simpler.

On page 7 in [1], Equation (9) becomes

$$
\begin{aligned}
\psi_{2 n}(x)= & \sum_{j=0}^{2 n} \alpha_{2 n-j} x^{2 n-j} \log x+\sum_{j=0}^{2 n} \beta_{2 n-j} x^{2 n-j}+ \\
& \sum_{p=0}^{n-1}(-1)^{p+1} \sum_{(\tau, \lambda) \in I_{p}} \sum_{\alpha \in S_{p, \tau, \lambda}^{\mathbb{R}}} \alpha^{-1}(\alpha+1)^{-1} \ldots(\alpha+2 n)^{-1} x^{\alpha+2 n}+ \\
& \sum_{p=0}^{n-1}(-1)^{p+1} \sum_{(\tau, \lambda) \in I_{p}} \sum_{\alpha \in S_{p, \tau, \lambda}^{-\rho+\lambda}} \alpha^{-1}(\alpha+1)^{-1} \ldots(\alpha+2 n)^{-1} x^{\alpha+2 n},
\end{aligned}
$$


where $0,-1, \ldots,-2 n \notin S_{p, \tau, \lambda}^{\mathbb{R}}, S_{p, \tau, \lambda}^{-\rho+\lambda}$ is the set of non-real singularities of $Z_{S}(s+\rho-\lambda, \tau)$, and $\alpha_{j}, \beta_{j}, j \in\{0,1, \ldots, 2 n\}$ are some explicitly computable constants.

The first inequality in [1] (p. 9) is now read as

$$
\begin{aligned}
\psi_{0}(x) \leq & \sum_{p=0}^{n-1}(-1)^{p+1} \sum_{\substack {(\tau, \lambda) \in I_{p} \\
\begin{subarray}{c}{\alpha \in S_{p, \tau, \lambda}^{\mathbb{R}} \\
0<\alpha \leq 2 \rho{ ( \tau , \lambda ) \in I _ { p } \\
\begin{subarray} { c } { \alpha \in S _ { p , \tau , \lambda } ^ { \mathbb { R } } \\
0 < \alpha \leq 2 \rho } }\end{subarray}} \alpha^{-1} x^{\alpha}+O\left(x^{2 \rho-1} d\right)+O\left(x^{\rho} K^{n-1}\right)+ \\
& O\left(d^{-2 n} x^{\rho+2 n} K^{-n-1}\right)+O(\log x) .
\end{aligned}
$$

The assertion follows by taking $d=x^{1-\frac{\rho}{n}}, K=x^{\frac{\rho}{n}}$.

Theorem 2. (Prime Geodesic Theorem) Let $Y$ be as above. Then

$$
\pi_{\Gamma}(x)=\sum_{p=0}^{n-1}(-1)^{p+1} \sum_{(\tau, \lambda) \in I_{p}} \sum_{\substack{\alpha \in S_{p}^{\mathbb{R}}, \tau, \lambda \\ 2 \rho-\frac{\rho}{n}<\alpha \leq 2 \rho}} \operatorname{li}\left(x^{\alpha}\right)+O\left(x^{2 \rho-\frac{\rho}{n}}(\log x)^{-1}\right),
$$

as $x \rightarrow \infty$, where $\pi_{\Gamma}(x)$ is the function counting prime geodesics on $Y$ of length not larger than $\log x$.

Proof. Follows immediately from Theorem 1.

Theorem 3. (Gallagherian Prime Geodesic Theorem) Let $Y$ be as above and $\varepsilon>0$. There exists a set $E$ of finite logarithmic measure such that

$$
\begin{aligned}
\pi_{\Gamma}(x)= & \sum_{p=0}^{n-1}(-1)^{p+1} \sum_{(\tau, \lambda) \in I_{p}} \sum_{\substack{\alpha \in S_{,, \tau, \lambda}^{\mathbb{R}} \\
2 \rho-\rho \frac{4 n+1}{4 n^{2}+1}<\alpha \leq 2 \rho}} \operatorname{li}\left(x^{\alpha}\right)+ \\
& O\left(x^{2 \rho-\rho \frac{4 n+1}{4 n^{2}+1}}(\log x)^{\frac{n-1}{4 n^{2}+1}-1}(\log \log x)^{\frac{n-1}{4 n^{2}+1}+\varepsilon}\right),
\end{aligned}
$$

as $x \rightarrow \infty, x \notin E$.

Proof. As a starting point, we take the explicit formula for $\psi_{2 n}(x)$ given by Equation (1).

Bearing in mind the fact that topological singularities are missing, and proceeding in the same way as in [1] (pp. 10-12), we conclude that for $x \notin E$ (Cf. [1] (p. 12, relation (21)))

$$
\begin{aligned}
\psi_{0}(x) \leq & \sum_{p=0}^{n-1}(-1)^{p+1} \sum_{\substack{(\tau, \lambda) \in I_{p} \\
\alpha \in S_{p, \tau, \lambda}^{\mathbb{R}} \\
0<\alpha \leq 2 \rho}} \alpha^{-1} x^{\alpha}+O\left(x^{2 \rho-1} d\right)+O\left(x^{\rho} Y^{n-1}\right)+ \\
& O\left(\frac{x^{\alpha}(\log x)^{\beta}(\log \log x)^{\beta+\varepsilon}}{d^{2 n}}\right)+O\left(d^{-2 n} \frac{x^{\rho+2 n}}{W^{n+1}}\right)+O(\log x) .
\end{aligned}
$$

The assertion of the theorem now follows by putting $d=x^{\frac{\alpha-2 \rho+1}{2 n+1}}(\log x)^{\frac{\beta}{2 n+1}}(\log \log x)^{\frac{\beta}{2 n+1}}, Y \sim x^{\frac{2 \rho+4 n-2 \alpha}{2 n+3}}(\log x)^{\frac{1-2 \beta}{2 n+3}}(\log \log x)^{\frac{1-2 \beta}{2 n+3}}$ into (2), $\alpha=\frac{8 n^{3}+2 n+\rho-6 n \rho}{4 n^{2}+1}, \beta=\frac{2 n^{2}-n-1}{4 n^{2}+1}$, and finalizing the argument in a standard way.

The author would like to thank the editor and anonymous referees who kindly reviewed the manuscript and provided valuable suggestions and comments. The authors apologize for any inconvenience caused and state that the scientific conclusions are unaffected. The original article has been updated. 


\section{References}

1. Gušić, D. Prime Geodesic Theorems for Compact Locally Symmetric Spaces of Real Rank One. Mathematics 2020, 8, 1762. [CrossRef]

2. Bunke, U.; Olbrich, M. Selberg Zeta and Theta Functions: A Differentiental Operator Approach; Akademie: Berlin, Germany, 1995. 